\title{
Radar observation of the Leonids in 1998 and 1999
}

\author{
M. Šimek and P. Pecina \\ Astronomical Institute of the Academy of Sciences of the Czech Republic, 25165 Ondřejov, Czech Republic \\ Received 26 June 2000 / Accepted 22 October 2000

\begin{abstract}
Results of Ondřejov radar observations of Leonid meteor shower in 1998 and 1999 are presented. It was found that while the activity in 1998 was dominated by the long-duration echoes resulting in a low mass distribution index averaged over an interval $23 \mathrm{~h}$ UT November 16 to $12 \mathrm{~h}$ UT November 17 as $s=1.23 \pm 0.02$ with the peak at $L_{\odot}=234.633 \pm 0.004$. The 1999 activity culminated at $L_{\odot}=235.285 \pm 0.002$ and was mainly due to prevailing of fainter echoes yielding a mean mass distribution index $s=1.58 \pm 0.05$ between $23 \mathrm{~h}$ UT November 17 $8 \mathrm{~h}$ UT November 18. The activity pattern in 1998 showed a multiple peak structure while the corresponding 1999 pattern showed only one distinct peak. The 1998 activity rates were lower than those in 1999 by the ratio of 2.8. Our observations indicate that the main outburst of this Leonid return occurred in 1999. The 1998 activity is characterized by a large scatter of observed hourly echo counts while the ones in 1999 possessed only one sharp maximum. The flux curve in 1998 shows multiple peak character while in 1999 the activity patterns of echoes with duration in excess of $0.4 \mathrm{~s}$ even though their peaks do not coincide generally with the activity maxima. The 1998 flux reached $3.3410^{-12} \mathrm{~m}^{-2} \mathrm{~s}^{-1}$ for a limiting mass of $m_{0}=10^{-5} \mathrm{~kg}$ while for 1999 it was determined as $37.4110^{-12} \mathrm{~m}^{-2} \mathrm{~s}^{-1}$.
\end{abstract}

Key words. comets: individual: 55P/Tempel-Tuttle - Leonids - meteors, meteoroids

\section{Introduction}

The Leonid meteor shower activity in 1998 and 1999 belong to most of the expressive events in the sky during the last decades of the 20th century. Due to favorable geometry conditions of the radiant and position of Ondřejov radar $\left(49^{\circ} 54^{\prime} 38^{\prime \prime} \mathrm{N}, 14^{\circ} 47^{\prime} 01^{\prime \prime} \mathrm{E}\right)$, it was possible to cover the main parts of shower activity in both years. The present results complete the picture of the events resulting from world-wide observations using all possible techniques.

\section{Observation}

The results of the Leonid meteor shower observations in 1998 and 1999 obtained at the Ondřejov observatory by a $20 \mathrm{~kW}$ meteor radar working at $37.5 \mathrm{MHz}$ are presented. The antenna is steerable in azimuth which enables reasonable observational results of the shower activity for the radiant zenith distances $z_{\mathrm{R}} \geq 15^{\circ}$.

\subsection{8}

The observations in 1998 were carried out on Nov. 17, 18, 19, and 20, always from $0 \mathrm{~h} \mathrm{LT} \mathrm{(23} \mathrm{h} \mathrm{UT)} \mathrm{till} 13 \mathrm{~h} \mathrm{LT}$

Send offprint requests to: P. Pecina, e-mail: ppecina@asu.cas.cz
(12 h UT). The antenna followed the intersection of $50^{\circ}$ almucantar with the $90^{\circ}$ circle centered at the shower radiant. For the radiant zenith angles less than $50^{\circ}$ the antenna was oriented to the azimuth differing from that of the shower radiant by $180^{\circ}$. The mean echo counts on 19 and 20 Nov. were considered to be the sporadic background activity. To obtain the shower echo rates mean sporadic echo counts in particular intervals were subtracted from recorded activity on Nov. 17.

\subsection{9}

The observations in 1999 were performed on Nov. 15, 17, 18 , and 19 between $0 \mathrm{~h}$ and $11 \mathrm{~h}$ local time ( $23 \mathrm{~h}-10 \mathrm{~h} \mathrm{UT})$. Contrary to 1998 the antenna followed $50^{\circ}, 40^{\circ}$, and $17^{\circ}$ almucantar intersection whenever possible. Otherwise the antenna was oriented to the azimuth differing from that of the shower radiant by $180^{\circ}$. The elevation of $50^{\circ}$ corresponds to maximum sensitivity of the radar while $17^{\circ}$ points to the maximum of the secondary lobe of the antenna. The mean echo rates observed on Nov. 15, 17, and 19 were considered as products of sporadic activity.

\section{Mass distribution}

Mass-distribution indices, $s$, were calculated from the slope of the linear dependence of a plot of the logarithmic cumulative number of shower echoes versus logarithmic 
Table 1. Mass-distribution index, $s$, in 1998 and 1999. Time indicates the beginning of a one-hour interval (UT). Results for 1998 refer to November 16/17, while those in 1999 are determined for November 17/18

\begin{tabular}{|c|c|c|c|c|c|c|}
\hline \multicolumn{7}{|c|}{ Time } \\
\hline \multirow{5}{*}{1998} & 1.195 & 1.222 & 1.245 & 1.156 & 1.206 & 1.176 \\
\hline & $\pm 0.011 \pm 0.009$ & \pm 0.010 & \pm 0.007 & \pm 0.010 & \pm 0.009 & \pm 0.010 \\
\hline & 07 & 08 & 09 & 10 & 11 & \\
\hline & $\begin{array}{ll}1.216 & 1.266\end{array}$ & 1.239 & 1.370 & 1.375 & 1.160 & \\
\hline & $\pm 0.008 \pm 0.008$ & \pm 0.015 & \pm 0.023 & \pm 0.017 & \pm 0.054 & \\
\hline \multicolumn{7}{|l|}{$\overline{\overline{\text { Time }}}$} \\
\hline \multirow[t]{6}{*}{1999} & 00 & 01 & 02 & 03 & 04 & 05 \\
\hline & 1.486 & 1.767 & 1.637 & 1.450 & 1.436 & 1.427 \\
\hline & $\pm 0.233 \pm 0.028$ & \pm 0.023 & \pm 0.012 & \pm 0.014 & \pm 0.020 & \pm 0.043 \\
\hline & 07 & 08 & & & & \\
\hline & $\begin{array}{ll}1.666 & 1.757\end{array}$ & 2.023 & & & & \\
\hline & $\pm 0.065 \pm 0.048$ & \pm 0.189 & & & & \\
\hline
\end{tabular}

echo duration. The index $s$ presented in Table 1 was calculated from cumulative counts, $N_{\mathrm{c}}$, of the shower echoes in 14 echo duration classes, $T$, within the interval of $0.4-$ $7.5 \mathrm{~s}$ according to the formula (Kaiser 1955)

$\log N_{\mathrm{c}}=-\frac{3}{4}(s-1) \log T+$ const.

It is apparent that $s$-values at the marginal periods in 1998 remain low even though the long duration echoes are not very frequent. We may conclude that the shower suffers from a lack of faint meteors. The complete distribution of shower echo durations is presented in Fig. 1. Extremely low $1998 s$-values can be compared with corresponding population index derived by Arlt \& Brown (1999) from world-wide visual observations of the Leonids 1998. Similar low $s$-values around 1.2 have been found also by Šimek \& Pecina (2000; hereafter Paper I) for Leonids 1965.

The Leonids 1999 are characterized by a different distribution of echo durations - see Table 1 and Fig. 2. Massdistribution indices, $s$, are generally higher which indicates a lower contribution from larger particles in the stream. This compares well with the visual population indices presented by Arlt et al. (1999).

\section{Activity profiles and flux}

Activity profiles as well as the flux of the shower were determined for the echo-duration intervals presented in Table 2.

\subsection{8}

Multiple simultaneous long duration echoes often appear on the film record, which could in some cases affect the number count of weak and short meteor echoes. Nevertheless, we are convinced that the record was not drastically saturated by long duration echoes which could
Table 2. The echo duration interval, corresponding radio magnitude interval, and interval of meteoroid mass, with $T$, $M_{\mathrm{r}}, M_{\infty}$ as their bound. $T$ is in seconds and $M_{\infty}$ in kilograms

\begin{tabular}{ccc}
\hline $\begin{array}{c}\text { Duration } \\
\text { interval }\end{array}$ & $\begin{array}{c}\text { radio magnitude } \\
\text { interval }\end{array}$ & $\begin{array}{c}\text { meteoroid mass } \\
\text { interval }\end{array}$ \\
\hline$T<0.4 \mathrm{~s}$ & $M_{\mathrm{r}}<+1.5$ & $M_{\infty}<5.710^{-6} \mathrm{~kg}$ \\
$T \geq 0.4 \mathrm{~s}$ & $M_{\mathrm{r}}>+1.5$ & $M_{\infty} \geq 5.710^{-6} \mathrm{~kg}$ \\
$T \geq 1.0 \mathrm{~s}$ & $M_{\mathrm{r}} \geq+0.7$ & $M_{\infty} \geq 1.510^{-5} \mathrm{~kg}$ \\
$T \geq 10 \mathrm{~s}$ & $M_{\mathrm{r}} \geq-1.3$ & $M_{\infty} \geq 1.910^{-4} \mathrm{~kg}$ \\
\hline
\end{tabular}

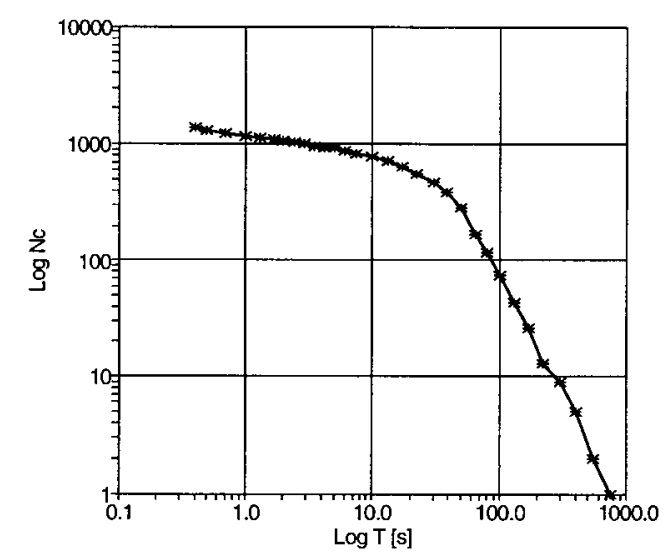

Fig. 1. $\log N_{\mathrm{c}}$ vs. $\log T$ fit of echoes with $T \geq 0.4 \mathrm{~s}$ for 1998

dramatically reduce the occurrence of shorter echoes. This is confirmed by the low values of mass-distribution indices, $s$, during whole observed shower interval, see Table 1.

Activity profiles of the shower were determined for echo-duration intervals presented in Table 2. The data have been calculated as described in Paper I but for the radiant zenith angle, $z_{\mathrm{R}}=48^{\circ}$, at $5 \mathrm{~h}$ UT. The profiles illustrating a dependence of recorded shower hourly rates on solar longitude, $L_{\odot}(\mathrm{J} 2000.0)$, are presented in Figs. $3-$ 10 . These rates are not corrected for the dependence response function of the radar on - the radiant zenith angle, antenna gain in the direction toward the reflection point, geocentric velocity of the shower, and the corresponding mass-distribution index. The real activity profile of the shower is apparent from the patterns of the flux discussed below.

The lack of faint shower meteors in 1998 having echo duration $T<0.4 \mathrm{~s}$ is evident. This group covers meteor echoes up to +8.5 radio magnitude. Shower activity is slightly pronounced only within a short interval $1 \mathrm{~h} 20 \mathrm{~m}-$ $6 \mathrm{~h} 20 \mathrm{~m}$. The other three categories of larger durations are characterized by very similar patterns which show a conglomerate of local activity peaking between $23 \mathrm{~h}$ Nov. 16 and $11 \mathrm{~h}$ Nov. 17, the position of which is practically identical. This is due to the dominant occurrence of the longduration echoes which produces low mass-distribution indices $s$ - see Table 1. All maxima are summarized in Table 3 . The patterns in Figs. $4-6$ show a broad activity interval which corresponds to the duration of the shower activity described by Arlt \& Brown (1999) from visual 
Table 3. Positions of activity peaks in 1998 and 1999 in four categories of echo durations are shown in Figs. 3-10. Since the flux has been determined only for echo duration $T \geq 0.4 \mathrm{~s}$ and $T \geq 1.0 \mathrm{~s}$, positions for $T<0.4 \mathrm{~s}$ and $T \geq 10 \mathrm{~s}$ are related to observed hourly echo rates. It is evident from Figs. 4, 5, 8, and 9 that observed echo rates before $3 \mathrm{~h} 10 \mathrm{~m}$ and after $5 \mathrm{~h} 50 \mathrm{~m}$ UT are affected by large correction for the antenna response function. It is apparent from Figs. 3, 6, 7, and 10 that the flux patterns in marginal periods would show well pronounced maxima, too

\begin{tabular}{|c|c|c|}
\hline \multirow{2}{*}{$\begin{array}{c}\text { Echo } \\
\text { duration }\end{array}$} & \multicolumn{2}{|c|}{17 November 1998} \\
\hline & $L_{\odot}[\mathrm{J} 2000.0]$ & Time $[\mathrm{UT}]$ \\
\hline \multirow{4}{*}{$T<0.4 \mathrm{~s}$} & $234^{\circ} .536 \pm 0.006$ & $2 \mathrm{~h} 05 \mathrm{~m} \pm 8 \mathrm{~m}$ \\
\hline & $234^{\circ} 633 \pm 0^{\circ} 004$ & $4 \mathrm{~h} 25 \mathrm{~m} \pm 6 \mathrm{~m}$ \\
\hline & $234.665 \pm 0.004$ & $5 \mathrm{~h} 10 \mathrm{~m} \pm 6 \mathrm{~m}$ \\
\hline & $234^{\circ} .707 \pm 0.007$ & $6 \mathrm{~h} 10 \mathrm{~m} \pm 10 \mathrm{~m}$ \\
\hline \multirow{5}{*}{$T \geq 0.4 \mathrm{~s}$} & $234^{\circ} .542 \pm 0.005$ & $2 \mathrm{~h} 15 \mathrm{~m} \pm 7 \mathrm{~m}$ \\
\hline & $234^{\circ} .567 \pm 0.007$ & $2 \mathrm{~h} 50 \mathrm{~m} \pm 10 \mathrm{~m}$ \\
\hline & $234.633 \pm 0.003$ & $4 \mathrm{~h} 25 \mathrm{~m} \pm 5 \mathrm{~m}$ \\
\hline & $234^{\circ} .658 \pm 0.004$ & $5 \mathrm{~h} 00 \mathrm{~m} \pm 6 \mathrm{~m}$ \\
\hline & $234^{\circ} .799 \pm 0.007$ & $8 \mathrm{~h} 22 \mathrm{~m} \pm 10 \mathrm{~m}$ \\
\hline$T \geq 1.0 \mathrm{~s}$ & \multicolumn{2}{|c|}{ the same as for $T \geq 0.4$ s echo duration group } \\
\hline$T \geq 10 \mathrm{~s}$ & \multicolumn{2}{|c|}{ the same as for $T \geq 0.4 \mathrm{~s}$ echo duration group } \\
\hline Echo & \multicolumn{2}{|c|}{18 November 1999} \\
\hline duration & $L_{\odot}[\mathrm{J} 2000.0]$ & Time $[\mathrm{UT}]$ \\
\hline$T<0.4 \mathrm{~s}$ & $235^{\circ} 290 \pm 0.002$ & $2 \mathrm{~h} 7.5 \mathrm{~m} \pm 2.5 \mathrm{~m}$ \\
\hline$T \geq 0.4 \mathrm{~s}$ & $235^{\circ} 285 \pm 0.002$ & $2 \mathrm{~h} 00 \mathrm{~m} \pm 2.5 \mathrm{~m}$ \\
\hline$T \geq 1.0 \mathrm{~s}$ & $235^{\circ} 287 \pm 0^{\circ} 003$ & $2 \mathrm{~h} 3.2 \mathrm{~m} \pm 3.7 \mathrm{~m}$ \\
\hline$T \geq 10 \mathrm{~s}$ & $235^{\circ} 297 \pm 0.002$ & $2 \mathrm{~h} 17.5 \mathrm{~m} \pm 2.5 \mathrm{~m}$ \\
\hline
\end{tabular}

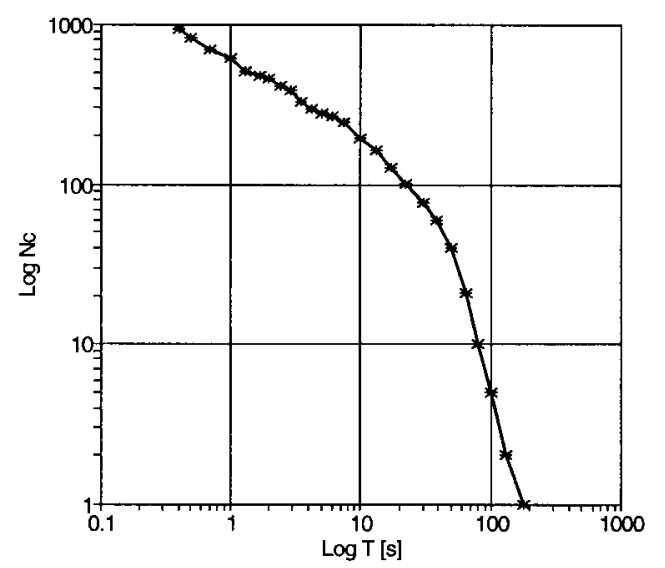

Fig. 2. $\log N_{\mathrm{c}}$ vs. $\log T$ fit of echoes with $T \geq 0.4 \mathrm{~s}$ for 1999

observations. Minor shower activity for mainly small particles was observed one day later, on 18 Nov.

\subsection{9}

The main feature of the shower is the dominant single activity peak in all four investigated shower echo categories presented in Figs. 7-10. Mass distribution indices and the positions of peaks are shown in Tables 1 and 3 . Maximum shower rates of echoes belonging to the first three categories in Table 3 appeared on $L_{\odot}=235.287$.

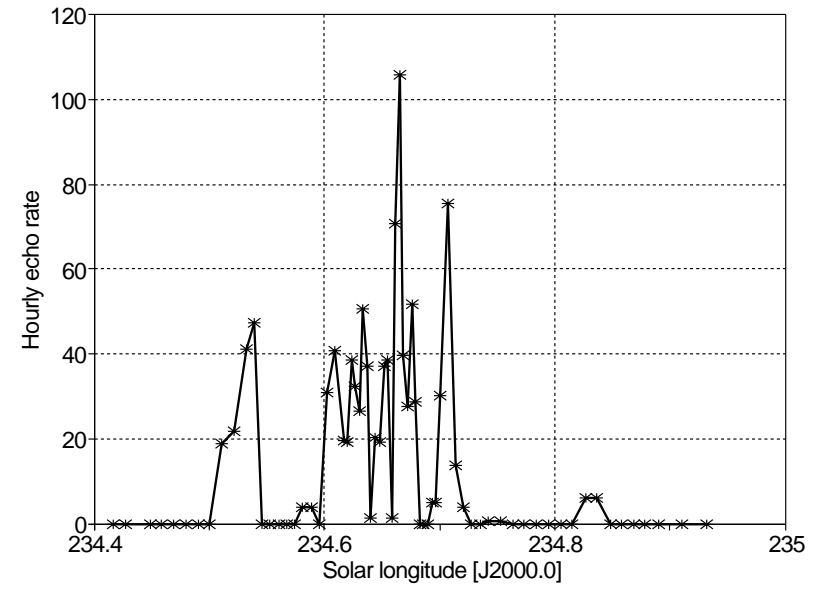

Fig. 3. Hourly echo rate of echoes with $T<0.4$ s for 1998

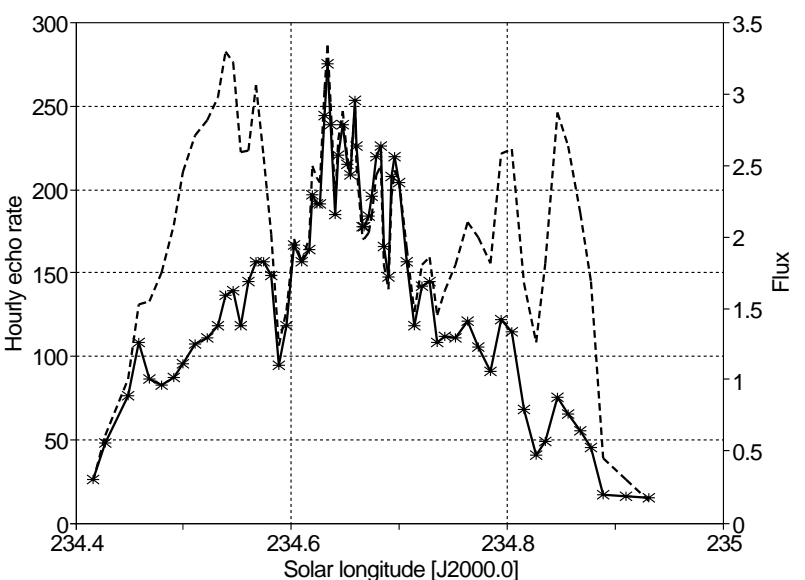

Fig. 4. Hourly echo rate of echoes with $T \geq 0.4 \mathrm{~s}$ (full line) and flux, $\Theta_{m_{0}}$, in $10^{-12} \mathrm{~m}^{-2} \mathrm{~s}^{-1}$ units, for 1998 (dashed line)

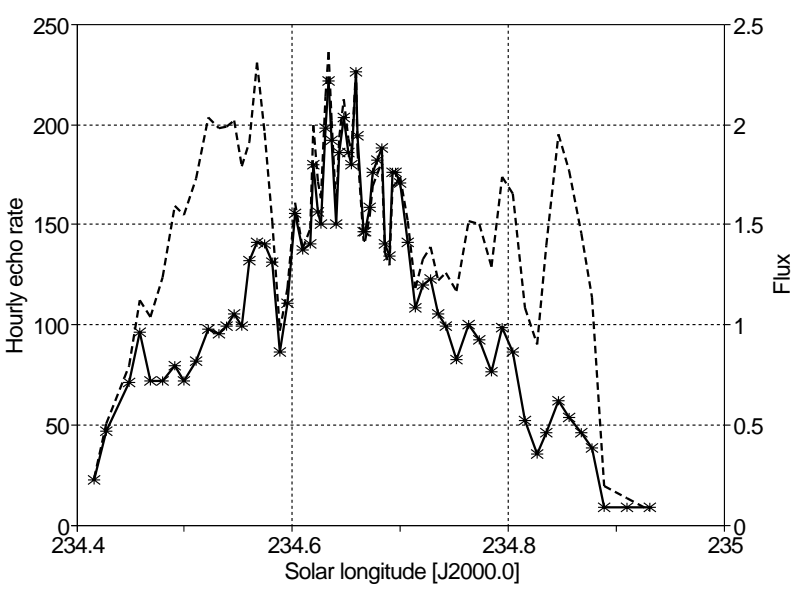

Fig. 5. The same as in Fig. 4 but for echoes with $T \geq 1 \mathrm{~s}$

The structure of the shower is quite different in comparison with 1998. The activity profiles of all categories are well pronounced showing a symmetrical patterns with the width for echo durations $T<0.4 \mathrm{~s}$ between half maximum of about 0.041 corresponding to $59 \mathrm{~min}$, and 0.026 (37 $\mathrm{min})$ for the other three categories. A similar width of the stream has been found by Arlt et al. (1999). 


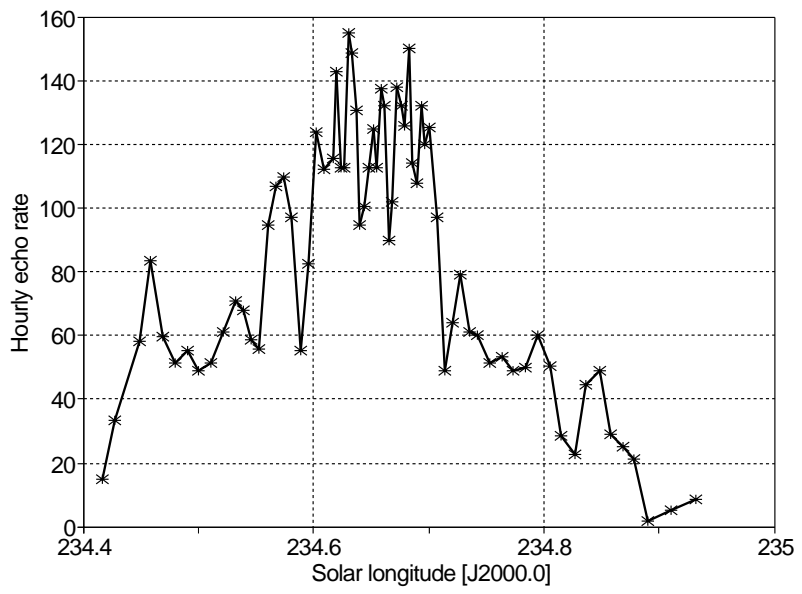

Fig. 6. The same as in Fig. 3 but for echoes with $T \geq 10 \mathrm{~s}$

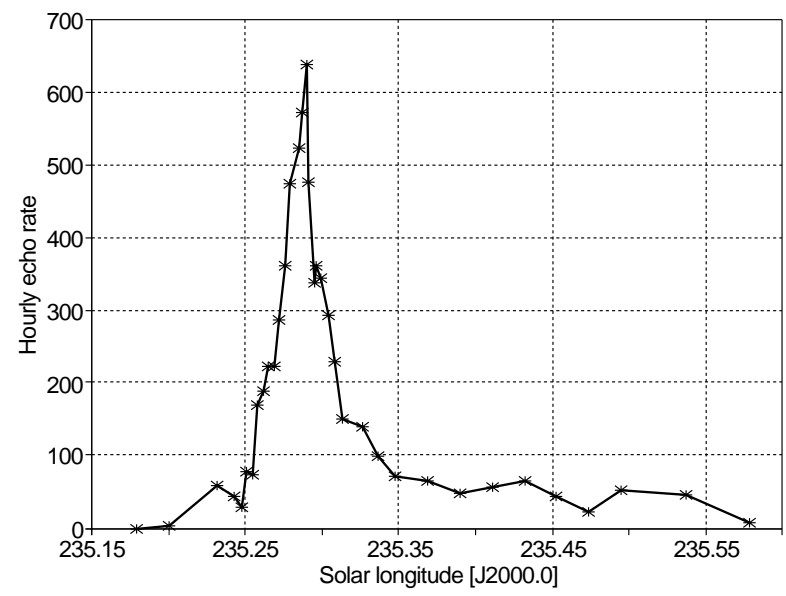

Fig. 7. Hourly echo rate of echoes with $T<0.4$ s for 1999

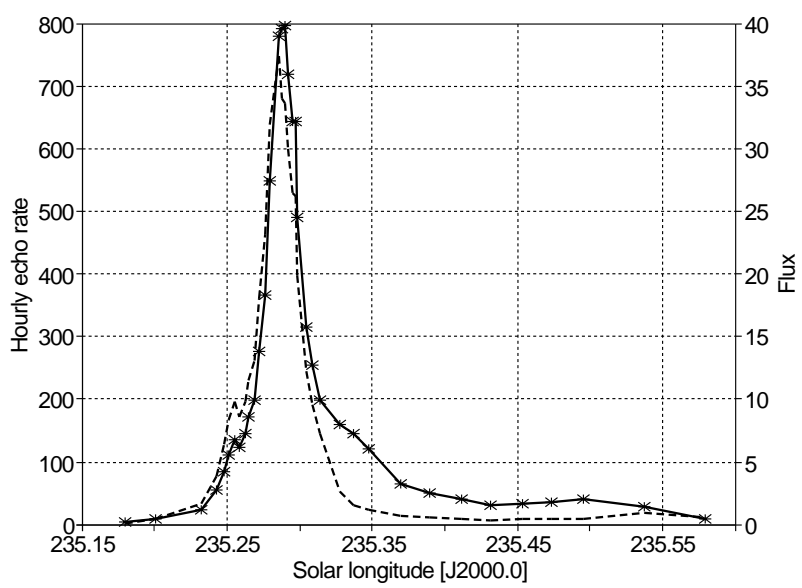

Fig. 8. Hourly echo rate of echoes with $T \geq 0.4 \mathrm{~s}$ (full line) and flux, $\Theta_{m_{0}}$, in $10^{-12} \mathrm{~m}^{-2} \mathrm{~s}^{-1}$ units, for 1999 (dashed line)

The activity peak of echoes having $T \geq 10 \mathrm{~s}$ was observed 15 minutes later at $L_{\odot}=235.297$. The higher contribution of faint meteors in the stream compared to 1998 is reflected in a higher index $s$ which is apparent also from Fig. 2.

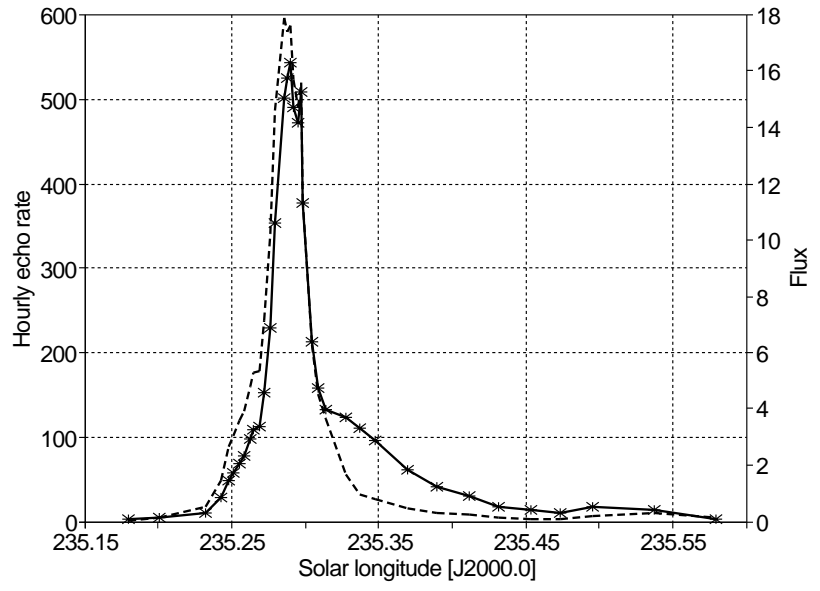

Fig. 9. The same as in Fig. 8 but for echoes with $T \geq 1 \mathrm{~s}$

\subsection{The flux}

The flux was computed according to Pecina (1982). Its values followed from the application of the formula relating flux to observed rates (e.g. Paper I). This article contains also some other information about the computing procedure and constants used. Here we add that the range limits of the integration in that formula depend on the particular angle, $\vartheta$, (measured clockwise), on the heights of beginning and of the end of the overdense part of an ionization curve corresponding to the duration of an echo and to the limits given by the radar equation requiring the minimum received power to be greater than about $210^{-13} \mathrm{~W}$ for Ondřejov radar. Mass distribution indices were taken from Table 1 for the whole particular hour. Other parameters needed for computation were the ablation parameter, $\sigma$, and shape-density coefficient, $K=A \Gamma \delta^{-2 / 3}$. The ablation parameter was set to $\sigma=0.1 \mathrm{~s}^{2} \mathrm{~km}^{-2}$ (Spurný et al. 2000) while $K=0.0597$ (in SI units), see Paper I. The flux values are related to $m_{0}=10^{-5} \mathrm{~kg}$ which is close to the lower value of the duration interval considered, and to $m_{0}=2.510^{-5} \mathrm{~kg}$ which is close to $T=1 \mathrm{~s}$. The flux related to the first mass is depicted in Figs. 4 and 8 while the flux related to the second mass is drawn in Figs. 5 and 9. The standard deviations of the flux values depend mainly on the standard deviations of the mass distribution index, $s$. The resulting relative standard deviations of the flux grouped around $10 \%$ of their values.

\section{Conclusions}

Leonid showers observed by the Ondřejov radar in 1998 and 1999 are characterized by quite different features of maximum activity expressed in corresponding solar longitude $L_{\odot}$, activity patterns, duration of the event, maximum values of the flux $\Theta_{m_{0}}$, and the distribution of shower particles along the Earth' path through the stream.

Comparing the positions of the maximum activities in 1998 with those for zenith hourly rates determined by Arlt \& Brown (1999), we see an agreement within 


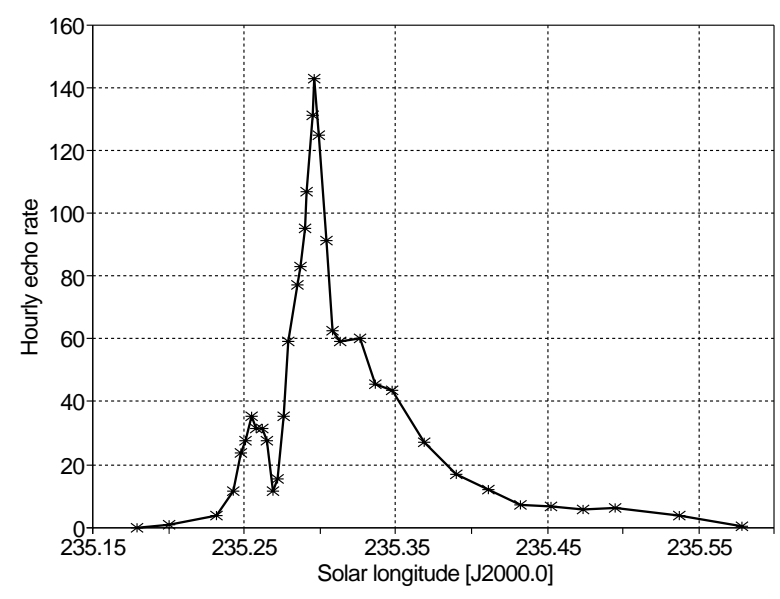

Fig. 10. The same as in Fig. 7 but for echoes with $T \geq 10 \mathrm{~s}$

derived errors of individual peaks at $L_{\odot}=234.536 \pm$ $0.006,234.633 \pm 0.004$, and $234.707 \pm 0.007$.

The flux curves for 1998 display many peaks and a large scatter which partly corresponds to the variability of corresponding echo hourly rates and partly to the variability of mass distribution indices as shown in Table 1. The maximum corresponds to the peak of activity and reached $\Theta_{m_{0}}=3.3410^{-12} \mathrm{~m}^{-2} \mathrm{~s}^{-1}$ for $m_{0}=10^{-5} \mathrm{~kg}$ at $4 \mathrm{~h} 25 \mathrm{~m} \pm 5 \mathrm{~m}$, and $\Theta_{m_{0}}=2.3610^{-12} \mathrm{~m}^{-2} \mathrm{~s}^{-1}$ for $m_{0}=2.510^{-5} \mathrm{~kg}$ at the same time. Visual and radar observations in 1998 show extremely low mass-distribution indices indicating a low activity of small particles in the stream followed by the dominant appearance of large particles. A similar effect was observed also in the Leonids 1965 - see McIntosh (1966), Plavcová (1968), McIntosh (1970), and Paper I.

Results for 1999 show high activity near $L_{\odot}=235.287$ (2 h 3 m UT) close to the 1966 position (cf. Paper I).
A similar location of the peak in 1999 was found by Arlt et al. (1999). The maximum hourly rate of the echo duration group $T \geq 10 \mathrm{~s}$ appeared 15 min later at $L_{\odot}=$ 235.297. The mass distribution index $s=1.58 \pm 0.05$ is comparable with a similar result in 1966 (cf. Paper I).

The flux curves for 1999 show (contrary to 1998) a well pronounced single maximum. The maximum flux reached the value $\Theta_{m_{0}}=37.4110^{-12} \mathrm{~m}^{-2} \mathrm{~s}^{-1}$ for $m_{0}=10^{-5} \mathrm{~kg}$ at $2 \mathrm{~h} 00 \mathrm{~m} \pm 2.5 \mathrm{~m}$ and $\Theta_{m_{0}}=17.9110^{-12} \mathrm{~m}^{-2} \mathrm{~s}^{-1}$ for $m_{0}=2.510^{-5} \mathrm{~kg}$ at the same time. The 1999 flux was found to exceed the 1998 values by slightly more than one order of magnitude.

Acknowledgements. This work was supported by the key project K1-003-601. The authors appreciate the help of Prof. W. J. Baggaley with the correction of the wording of the article.

\section{References}

Arlt, R., \& Brown, P. 1999, WGN, 27, 267

Arlt, R., Rubio, L. B., Brown, P., \& Gyssens, M. 1999, WGN, 27,286

Kaiser, T. R. 1955, in Meteors, ed. T. R. Kaiser (Pergamon Press, London), 119

McIntosh, B. A. 1966, Can. J. Phys., 44, 2729

McIntosh, B. A. 1970, in Evolutionary and Physical Properties of Meteoroids, ed. C. L. Hemenway, P. M. Millman, \& A. F. Cook, NASA SP-319, Washington, D.C, 193

Pecina, P. 1982, Bull. Astron. Inst. Czechosl., 33, 1

Plavcová, Z. 1968, in Physics and Dynamics of Meteors, ed. L'. Kresák, \& P. M. Millman (D. Reidel Publ. Co, Dordrecht), 432

Spurný, P., Betlem, H., van`t Leven, J., \& Jenniskens, P. 2000, Meteor. Planet. Sci., 35, 243

Šimek, M., \& Pecina, P. 2000, A\&A, 357, 777 